\title{
3-戊烯腈在零价镍催化体系中的平衡反应
}

\author{
董建勋 ${ }^{a, b}$ 石玉坤 ${ }^{a}$ 胡晓静 ${ }^{a}$ 冯晓燕 ${ }^{b}$ 宋娟娟 ${ }^{a}$ 丁真贞 $^{a}$ \\ 刘向明 $a$ 黄唯平 $* a$ \\ ( ${ }^{a}$ 南开大学化学系 天津 300071) \\ $\left({ }^{b}\right.$ 中国平煤神马能源化工集团尼龙化工公司 平顶山 467013)
}

\begin{abstract}
摘要 在探索 3-戊烯腈氢氰化合成已二腈反应的机理过程中, 发现 3-戊烯腈在零价镍体系中可能同时存在多个平衡反 应, 在不外加氧化氢条件下, 3-戊烯腈可以生成甲基戊二腈和已二腈. 采用零价镍、膦配体和无水 $\mathrm{ZnCl}_{2}$ 组成的催化体 系研究了该反应过程, 考察了反应温度和配体的影响, 并采用原位红外对反应进行监测, 提出了可能的反应机理.

关键词＼cjkstart零价镍; 3-戊烯腈; 2-甲基戊二腈; 己二腈; 反应平衡
\end{abstract}

\section{Nickel(0)-Catalyzed Equilibrium Reaction of 3-Pentenenitrile}

\author{
Dong, Jianxun ${ }^{a, b} \quad$ Shi, Yukun $^{a} \quad \mathrm{Hu}$, Xiaojing $^{a} \quad$ Feng, Xiaoyan $^{b} \quad$ Song, Juanjuan ${ }^{a}$ \\ Ding, Zhenzhen ${ }^{a} \quad$ Liu, Xiangming ${ }^{a} \quad$ Huang, Weiping ${ }^{*, a}$ \\ ( ${ }^{a}$ College of Chemistry, Nankai University, Tianjin 300071) \\ ( ${ }^{b}$ China Pingmei Shenma Energy and Chemical Group Nylon Chemical Company, Pingdingshan 467013)
}

\begin{abstract}
The equilibrium reaction of 3-pentenenitrile was carried out in the system containing Ni(0), P-ligand and anhydrous $\mathrm{ZnCl}_{2}$. The equilibrium state of 3-pentenenitrile was affected by factors such as reaction temperatures and different ligands, which were investigated. The FTIR-ATR was used to monitor the reaction process of 3-pentenenitrile turning into 2-methylglutaronitrile and adiponitrile in absence of HCN and the possible reaction mechanism was proposed.

Keywords zero-valent nickel; 3-pentenenitrile; 2-methylglutaronitrile; adiponitrile; reaction equilibrium
\end{abstract}

己二腈(ADN)主要用于生产已二胺(尼龙 66 的主要 原料之一)及其它精细化工和有机合成领域. 目前, 已二 腈的合成主要采用杜邦公司于 20 世纪 70 年代开发的丁 二烯直接氰化法, 该方法主要分为三步 ${ }^{[1 \sim 6]}$, 其中第三 步反应不仅是整个反应的决速步骤 $[7,8]$, 也对整个反应 过程的总选择性有较大影响(Scheme 1). 在已报道的有 关 2-甲基-3-丁烯腈(2M3BN)异构化为 3-戊烯腈(3PN) 的文献中, 零价镍催化体系主要是由环辛二烯镍和含磷

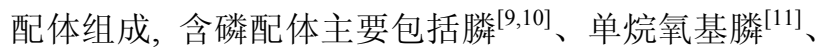
磷酸酯 ${ }^{[12]}$ 和亚磷酸酯 ${ }^{[13]}$ 等. 近来出现该反应在水溶液 ${ }^{[14]}$ 和离子液体 ${ }^{[15]}$ 中进行的报道. 所有关于 $2 \mathrm{M} 3 \mathrm{BN}$ 异构为 $3 \mathrm{PN}$ 的零价镍催化的反应过程中, 都产生了中间体 $\pi$-烯 丙基镍化合物 ${ }^{[16]}$.

关于 $3 \mathrm{PN}$ 在催化体系中的存在状态和反应迄今没 有相关的报道或者没受到重视. 为了研究探索 $3 \mathrm{PN}$ 在零

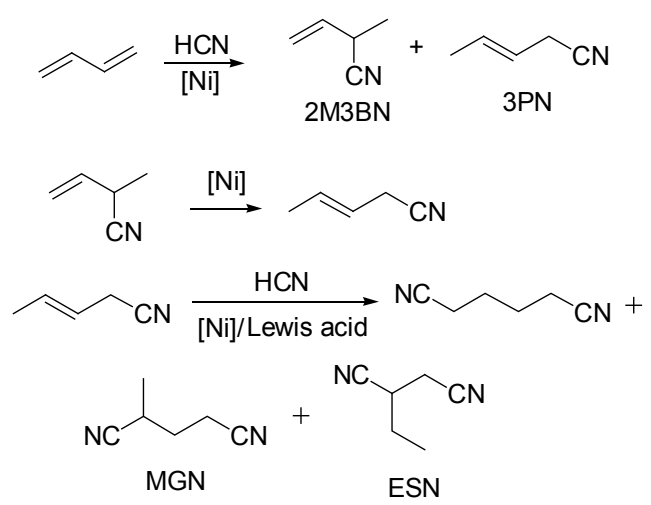

Scheme 1

价镍催化体系中可能存在的平衡反应，本文利用零价 镍、含磷配体和无水 $\mathrm{ZnCl}_{2}$ 组成催化体系开展了实验工

\footnotetext{
*E-mail: huangw@eyou.com

Received July 20, 2011; revised October 25, 2011; accepted November 4, 2011.

Project supported by the National Natural Science Foundation of China (No. 21071086).

国家自然科学基金(No. 21071086)资助项目.
} 
作. 研究发现: 在不外加 $\mathrm{HCN}$ 的条件下, $3 \mathrm{PN}$ 可以直接 生成 2-甲基戊二腈(MGN)和 $\mathrm{ADN}$, 同时还可能生成少 量的苯甲腈(BN), 反应温度和配体对该反应过程有重要 影响. 用原位红外对反应进行监测, 对反应提出了可能 的反应机理.

\section{1 实验部分}

\section{1 仪器和试剂}

实验仪器: 美国 Thermo Finnigan 公司 Polaris-Q气 相色谱质谱联用仪; 瑞士 Mettler Toledo Autochem 化学 公司 React IR ic10 原位红外仪; 北京北分瑞利分析仪器 公司 $3420 \mathrm{~A}$ 气相色谱仪.

实验试剂: 无水 $\mathrm{NiCl}_{2}$ 、苯甲腈 $(\mathrm{BN})$ 、锌粉、无水 $\mathrm{ZnCl}_{2}$ 、三苯膦 $\left(\mathrm{PPh}_{3}\right) 、 1,2-$ 双 (二苯基膦)乙烷(DPPE)、 1,2-双(二苯基膦)丁烷(DPPB)等均购于北京百灵威试剂 有限公司; 3-戊烯腈(3PN)和己二腈(ADN)购于西格玛奥 德里奇(上海)贸易有限公司; 2-甲基戊二腈(MGN)购于 上海迈瑞尔化学技术有限公司.

\section{2 实验过程}

连接无水无氧体系, 在 $50 \mathrm{~mL}$ 三颈反应瓶中放入磁 子, 插上温度计和冷凝管, 反应瓶置于磁力搅拌油浴加 热装置中. 在氩气保护下分别加入 $0.20 \mathrm{~g}$ 无水 $\mathrm{NiCl}_{2}$ 、一 定量的含磷配体和 $0.2 \mathrm{~g} \mathrm{ZnCl}_{2}$, 密封反应瓶, 氩气置换 $10 \mathrm{~min}$ 后用注射器加入 $5 \mathrm{~mL}$ 干燥 $3 \mathrm{PN}$, 升温至 $110{ }^{\circ} \mathrm{C}$ 搅拌 $1 \mathrm{~h}$; 降至室温后加入 $0.10 \mathrm{~g} \mathrm{Zn}$ 粉, 继续摚拌 $1 \mathrm{~h}$, 取样后升温至一定反应温度, 维持 $4 \mathrm{~h}$. 定时取样, 样品 用气相色谱跟踪分析 ${ }^{[17]}$, 壬烷为内标物.

\section{3 样品分析}

气相色谱条件: 气相色谱毛细管柱 SE-30 (50 $\mathrm{m} \times$ $0.53 \mathrm{~mm} \times 0.25 \mu \mathrm{m})$, 汽化室温度 $270{ }^{\circ} \mathrm{C}$, 检测器温度 $275{ }^{\circ} \mathrm{C}$, 载气氮气(纯度大于 $99.99 \%$ )压力 $200 \mathrm{kPa}$, 氢气 压力 $200 \mathrm{kPa}$, 空气压力 $500 \mathrm{kPa}$. 升温程序:

$$
80{ }^{\circ} \mathrm{C}(1 \mathrm{~min}) \stackrel{5^{\circ} \mathrm{C} / \mathrm{min}}{\longrightarrow} 100^{\circ} \mathrm{C} \stackrel{25^{\circ} \mathrm{C} / \mathrm{min}}{\longrightarrow} 230{ }^{\circ} \mathrm{C}(5 \mathrm{~min})
$$

质谱条件: Polaris-Q Thermo Finnigan 美国; 气相色谱毛
细管柱 HP-5 $(30 \mathrm{~m} \times 0.25 \mathrm{~mm} \times 0.25 \mu \mathrm{m})$, 汽化室温度 $240{ }^{\circ} \mathrm{C}$, 离子源温度 $240{ }^{\circ} \mathrm{C}$, 离子阱温度 $200{ }^{\circ} \mathrm{C}$; 电离 源: EI, $70(1 \mathrm{~min}) \sim 230{ }^{\circ} \mathrm{C}, 15{ }^{\circ} \mathrm{C} / \mathrm{min}$.

\section{2 结果与讨论}

\section{1 温度的影响}

我们首先考察了不同温度对 3PN 平衡反应的影响, 表 1 给出了不同温度下平衡时 $\mathrm{BN}, \mathrm{MGN}$ 和 $\mathrm{ADN}$ 的浓 度. 从表 1 可以看出, 随着温度从 $80{ }^{\circ} \mathrm{C}$ 升至 $140{ }^{\circ} \mathrm{C}$ 的 过程中，三种产物的浓度都逐渐增加，但是 $\mathrm{MGN}$ 的增 幅最大，产物浓度从 $152.3 \mathrm{mmol} \cdot \mathrm{L}^{-1}$ 增加为 346.4 $\mathrm{mmol} \cdot \mathrm{L}^{-1}$. 由此可以看出, 高温对 $3 \mathrm{PN}$ 的平衡反应影响 较大, 温度升高时有利于生成 3 种产物, 更易生成 MGN.

表 1 不同温度对 $3 \mathrm{PN}$ 平衡反应的影响 ${ }^{a}$

Table 1 Effect of various temperatures on the title reaction

\begin{tabular}{ccccc}
\hline Entry & $\begin{array}{c}\text { Temperature/ } \\
{ }^{\circ} \mathrm{C}\end{array}$ & $\begin{array}{c}\mathrm{BN} / \\
\left(\mathrm{mmol} \bullet \mathrm{L}^{-1}\right)\end{array}$ & $\begin{array}{c}\mathrm{MGN} / \\
\left(\mathrm{mmol} \bullet \mathrm{L}^{-1}\right)\end{array}$ & $\begin{array}{c}\mathrm{AND} / \\
\left(\mathrm{mmol} \bullet \mathrm{L}^{-1}\right)\end{array}$ \\
\hline 1 & 80 & 0.3871 & 152.3 & 19.69 \\
2 & 110 & 1.818 & 193.6 & 41.92 \\
3 & 125 & 4.640 & 234.5 & 61.51 \\
4 & 140 & 9.985 & 346.4 & 103.9 \\
\hline
\end{tabular}

${ }^{a} 5 \mathrm{~mL}$ of $3 \mathrm{PN}, 0.20 \mathrm{~g}$ of $\mathrm{NiCl}_{2}, 0.20 \mathrm{~g}$ of $\mathrm{ZnCl}_{2}, 0.10 \mathrm{~g}$ of $\mathrm{Zn}$ power, $\mathrm{Ni} / \mathrm{PPh}_{3} / \mathrm{ZnCl}_{2}$ molar ratio $1: 5: 1,4 \mathrm{~h}$.

\section{2 配体的影响}

不同配体存在空间位阻和电荷效应 ${ }^{[18]}$, 表 2 列出了 不同配体对 3PN 平衡反应的影响. 从表 2 可以看出, 配 体对 3 种产物的浓度有较大的影响。在 DPPB, $\mathrm{P}\left(\mathrm{OC}_{6} \mathrm{H}_{5}\right)_{3}$ 和 $\mathrm{P}\left(\mathrm{OCH}_{3}\right)_{3}$ 三种配体中, DPPB 的催化效果最 好, 平衡时 3 种产物的浓度最高, 可能原因是电荷效应 造成的; 而在 DPPB, DPPE 和 $\mathrm{PPh}_{3}$ 三配体中, DPPB 的 催化效果最好, 造成这种结果的可能原因是配体结构性 质差异 $(\text { 如 } \mathrm{P}-\mathrm{Ni}-\mathrm{P} \text { 键角 })^{[19,20]}$ ，即空间位阻的不同引起 的. 值得注意的是, 当催化体系使用 DPPB, DPPE 和 $\mathrm{PPh}_{3}$ 三配体时，平衡产物中有 $\mathrm{BN}$ 生成; 而用 $\mathrm{P}\left(\mathrm{OC}_{6} \mathrm{H}_{5}\right)_{3}$ 和 $\mathrm{P}\left(\mathrm{OCH}_{3}\right)_{3}$ 二种配体时, 产物中没有 $\mathrm{BN}$ 生成. 这意味 着反应过程中，配体有键的断裂.

表 2 不同配体的影响 ${ }^{a}$

Table 2 Effect of different ligands on the title reaction

\begin{tabular}{clcccc}
\hline Entry & Ligand & $\mathrm{Ni} / \mathrm{PPh}_{3}($ molar ratio $)$ & $\mathrm{BN} /\left(\mathrm{mmol} \cdot \mathrm{L}^{-1}\right)$ & $\mathrm{MGN} /\left(\mathrm{mmol} \cdot \mathrm{L}^{-1}\right)$ & $\mathrm{ADN} /\left(\mathrm{mmol} \cdot \mathrm{L}^{-1}\right)$ \\
\hline 1 & $\mathrm{DPPB}$ & $1: 2.5$ & 1.465 & 294.1 & 98.70 \\
2 & $\mathrm{DPPE}$ & $1: 2.5$ & 0.2956 & 3.551 & 1.048 \\
3 & $\mathrm{PPh}_{3}$ & $1: 5$ & 1.818 & 193.6 & 41.92 \\
4 & $\mathrm{P}\left(\mathrm{OC}_{6} \mathrm{H}_{5}\right)_{3}$ & $1: 5$ & 0 & 3.279 & 0.5321 \\
5 & $\mathrm{P}\left(\mathrm{OCH}_{3}\right)_{3}$ & $1: 5$ & 0 & 0.8101 & 0.0480 \\
\hline
\end{tabular}

${ }^{a} 5 \mathrm{~mL}$ of $3 \mathrm{PN}, 0.20 \mathrm{~g}$ of $\mathrm{NiCl}_{2}, 0.20 \mathrm{~g}$ of $\mathrm{ZnCl}_{2}, 0.10 \mathrm{~g}$ of $\mathrm{Zn}$ power, $110{ }^{\circ} \mathrm{C}, 4 \mathrm{~h}$. 


\section{3 零价镍与配体比的影响}

表 3 给出了不同镍/含磷配体物质的量的比对反应 的影响. 由于零价镍与四个配体配位才能达到 18 电子 稳定结构, 参加反应时需要失去一个配体才能与反应底 物结合, 因此镍与配体存在平衡反应. 零价镍是由二价 镍盐还原并与配体结合生成, 从表 3 可以看出, 镍与配 体的物质的量的比从 $1: 3$ 变化到 $1: 5$ 时, BN, MGN 和 $\mathrm{ADN}$ 在反应液中的浓度都有所增加. 可能原因是配体 量在一定范围内增加时, 提高了零价镍的生成量, 从而 导致了三种产物浓度的提高. 但配体量增加超过一定范 围时, 三种产物的浓度没有明显提高, 可能原因是零价 镍配位能力已达到最大量, 所以继续增加配体时三种产 物的浓度没有明显增加.

表 3 不同镍膦配体比的影响 ${ }^{a}$

Table 3 Effect of $\mathrm{Ni} / \mathrm{PPh}_{3}$ molar ratio on the title reaction

\begin{tabular}{ccccc}
\hline Entry & $\mathrm{Ni} / \mathrm{PPh}_{3}$ & $\begin{array}{c}\mathrm{BN} / \\
\left(\mathrm{mmol} \bullet \mathrm{L}^{-1}\right)\end{array}$ & $\begin{array}{c}\mathrm{MGN} / \\
\left(\mathrm{mmol} \bullet \mathrm{L}^{-1}\right)\end{array}$ & $\begin{array}{c}\mathrm{ADN} / \\
\left(\mathrm{mmol} \bullet \mathrm{L}^{-1}\right)\end{array}$ \\
\hline 1 & $1: 5$ & 1.818 & 193.6 & 41.92 \\
2 & $1: 4$ & 1.738 & 145.9 & 34.07 \\
3 & $1: 3$ & 1.567 & 111.1 & 22.56 \\
\hline
\end{tabular}

\section{4 反应机理讨论}

对于 3PN 异构化并进一步和氧化氢反应生成 ADN 的反应机理, 目前普遍认为 $3 \mathrm{PN}$ 异构化生成的 4PN 与 $\mathrm{HNiL}_{2} \mathrm{CN}$ (16 电子)作用, 通过镍与 $4 \mathrm{PN}$ 的 $\pi$ 键结合产生 新的催化中间体, 后者发生分子内 $\mathrm{H}$ 迁移, 将 $\mathrm{H}$ 加到 $\pi$ 键上, 还原形成 $\mathrm{C}-\mathrm{Ni}$ 键合 ${ }^{[21]}$. 进一步引入自由配体、 发生还原消除, 生成产物 $\mathrm{ADN}$ 和副产物 MGN, 同时重 新形成催化剂 $\mathrm{NiL}_{3}$, 继续进行氢氰化的循环反应. 在这 一过程中, 定向加成反应是全过程成败的关键, 否则将 生成大量 MGN 和乙基丁二腈(ESN)等副产物, 丁二烯 氢氰化法合成 ADN 关键性定向加成反应机理如 Scheme $2^{[22]}$.

结合现有文献和以上的研究结果, 我们推测 $3 \mathrm{PN}$ 在 零价镍催化体系中存在多种反应平衡, Scheme 3 中列出 了 3PN 可能存在的所有平衡反应. 在不外加 $\mathrm{HCN}$ 的条 件下, 3PN 生成 MGN 和 ADN, 可以确定反应 1, 2 和 5 存在, 因为只有反应 1 微观可逆向生成 HCN 和丁二烯 方向移动时才能提供氧源，反应 2 生成 $4 \mathrm{PN}$ ，反应 5 生 成 ADN. 分析 $3 \mathrm{PN}$ 在零价镍的反应液, 我们发现很少

${ }^{a} 5 \mathrm{~mL}$ of $3 \mathrm{PN}, 0.20 \mathrm{~g}$ of $\mathrm{NiCl}_{2}, 0.20 \mathrm{~g}$ of $\mathrm{ZnCl}_{2}, 0.10 \mathrm{~g}$ of $\mathrm{Zn}$ power, $4 \mathrm{~h}$.

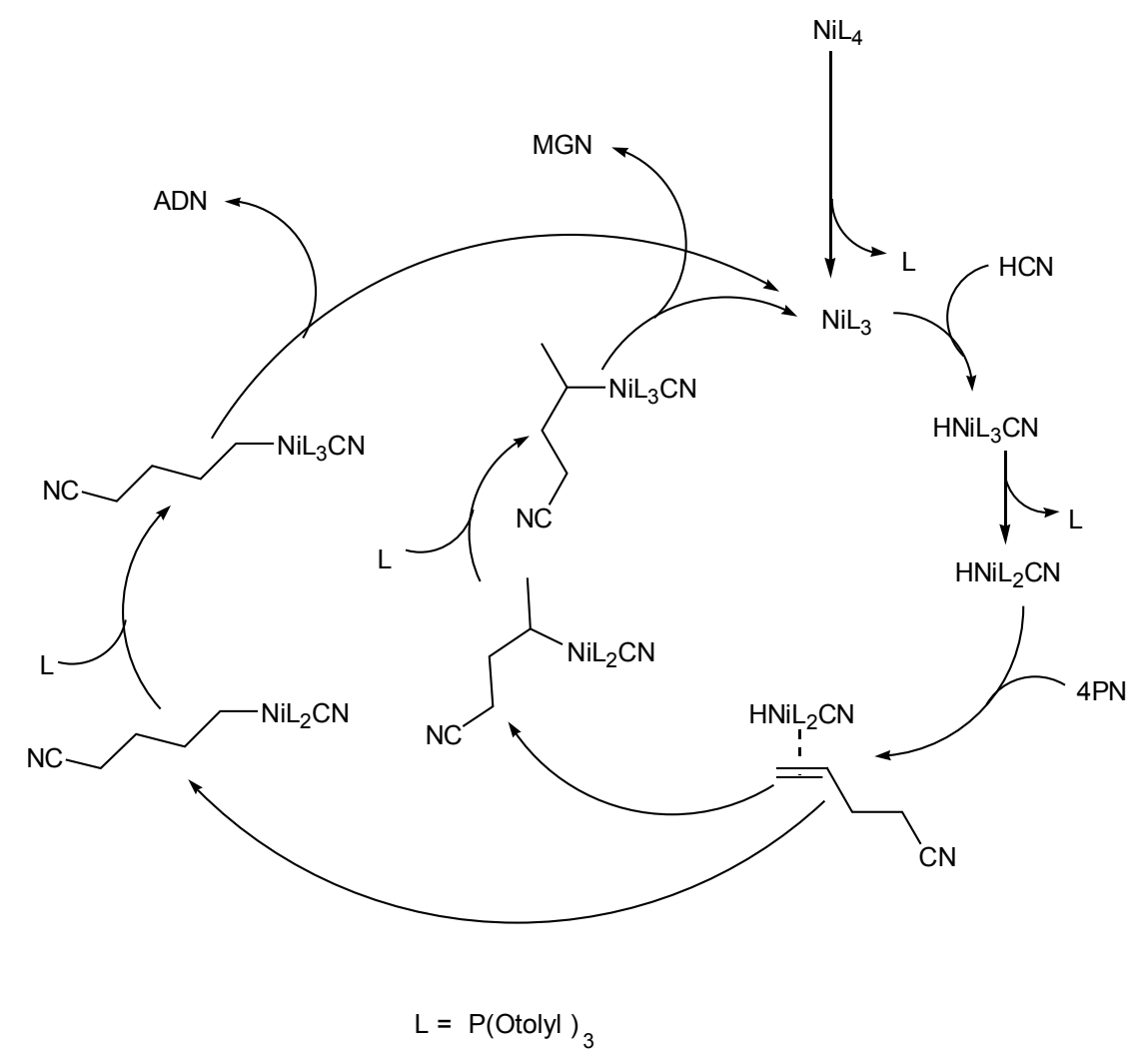

ADN/MGN $=3.3$ with no promoter

Scheme 2 


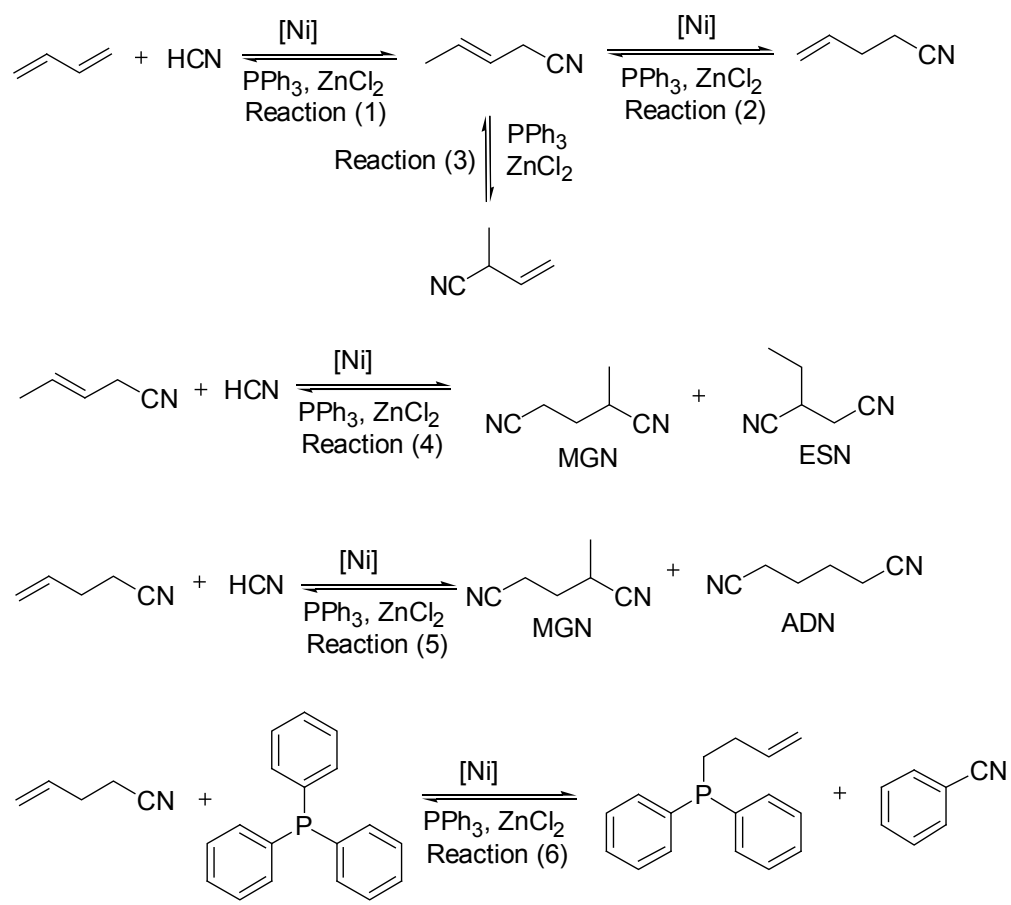

Scheme 3

量的 2M3BN, 2M2 $\mathrm{BN}$ 和 $2 \mathrm{PN}$, 其中 $2 \mathrm{PN}$ 是 $3 \mathrm{PN}$ 异构为 3 存在, 很少量存在的原因可能是因为 $3 \mathrm{PN}$ 的相对能量 比 $2 \mathrm{M} 3 \mathrm{BN}$ 低 $-9 \mathrm{~kJ} / \mathrm{mol}^{[23]}$, Scheme 4 列出了 $3 \mathrm{PN}$ 的几种 同分异构体的相对能量对比.

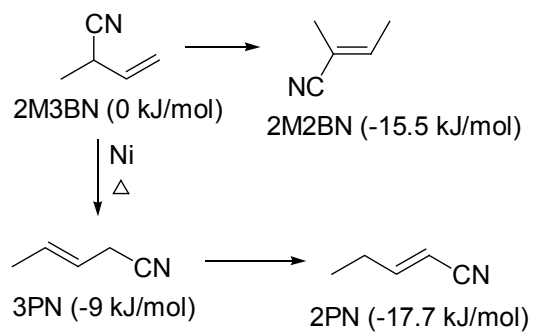

\section{Scheme 4}

反应 4 在 $3 \mathrm{PN}$ 氢氰化反应过程中已确定存在, 在 Scheme 1 中已经说明, 在反应液中没有检测到 ESN, 因 此可能主要发生反应 5. 我们在反应液中色谱检测到有 BN 生成, 通过质谱检测还有 3-丁烯基二苯基膦生成, 因此确定存在反应 6 . 由表 2 可以看出, 配体为膦类时, 有 $\mathrm{BN}$ 生成; 配体为亚磷酸酯时, 没有 $\mathrm{BN}$ 生成, 可以进 一步证明 BN 是通过反应 6 生成的.

为了进一步探索反应机理, 我们采用原位红外仪监 测了反应过程. 图 1 显示了反应过程中反应物中 $\mathrm{CN}$ 基 吸收峰的变化情况. 3PN 中的氧基吸收峰在 2248 $\mathrm{cm}^{-1}{ }^{[24]}$, 而 BN, MGN 和 ADN 的氰基吸收峰在 2220 $2260 \mathrm{~cm}^{-1}[25]$ 之间. 图 1a 中 $2248 \mathrm{~cm}^{-1}$ 的吸收峰随着反 应进行逐渐减弱, 而 $2230 \mathrm{~cm}^{-1}$ 的吸收峰则逐渐加强,
$4 \mathrm{PN}$ 时的副产物，而 $2 \mathrm{M} 3 \mathrm{BN}, 2 \mathrm{M} 2 \mathrm{BN}$ 的产生表明反应 图 $1 \mathrm{~b}$ 可以更清楚的看出 $2230 \mathrm{~cm}^{-1}$ 的吸收峰随着反应时 间延长而逐渐增加的过程. 同时，我们推测 $2230 \mathrm{~cm}^{-1}$ 的 $\mathrm{CN}$ 基吸收峰是 $\mathrm{MGN}, \mathrm{ADN}$ 和 BN 中一种或多种产物 的 CN 基吸收峰. 从气相色谱图和质谱图(辅助材料)来 看, MGN 在所有产物中的比例较大, $2230 \mathrm{~cm}^{-1}$ 的吸收 峰可能主要是 MGN 的中的 $\mathrm{CN}$ 基吸收峰引起的. 因此, 可以证明, 3PN 在零价镍体系中可以在不加 $\mathrm{HCN}$ 的条件 下可以生成 $\mathrm{MGN}, \mathrm{ADN}$ 和 BN 中的一种或多种产物.

\section{3 结论}

通过研究我们发现, 3PN 在零价镍催化体系中同时 存在多个平衡反应，在不外加氧化氢条件下，3PN 可以 生成 MGN、ADN 和 $\mathrm{BN}$ ，反应温度和配体对该反应过 程有较大的影响, 温度为 $140^{\circ} \mathrm{C}$ 和配体为 $\mathrm{DPPB}$ 时最有 利于三者生成. 从整个反应过程来看三种产物可能的生 成路径, 生成 $1 \mathrm{~mol}$ 的 MGN 或 ADN, 需要消耗 $2 \mathrm{~mol}$ 的 3PN，这降低了目标产物的收率，因此需要抑制这些反 应的发生.如何控制这些反应，有待进一步研究.

辅助材料(Supporting Information) 3PN 在零价镍催 化体系中样品的气相色谱和质谱图. 这些材料可以免费 从本刊网站(http://sioc-journal.cn/)上下载.

致谢 原位红外实验和质谱分析得到了南开大学元素 有机化学国家重点实验室的大力支持, 在此表示感谢. 

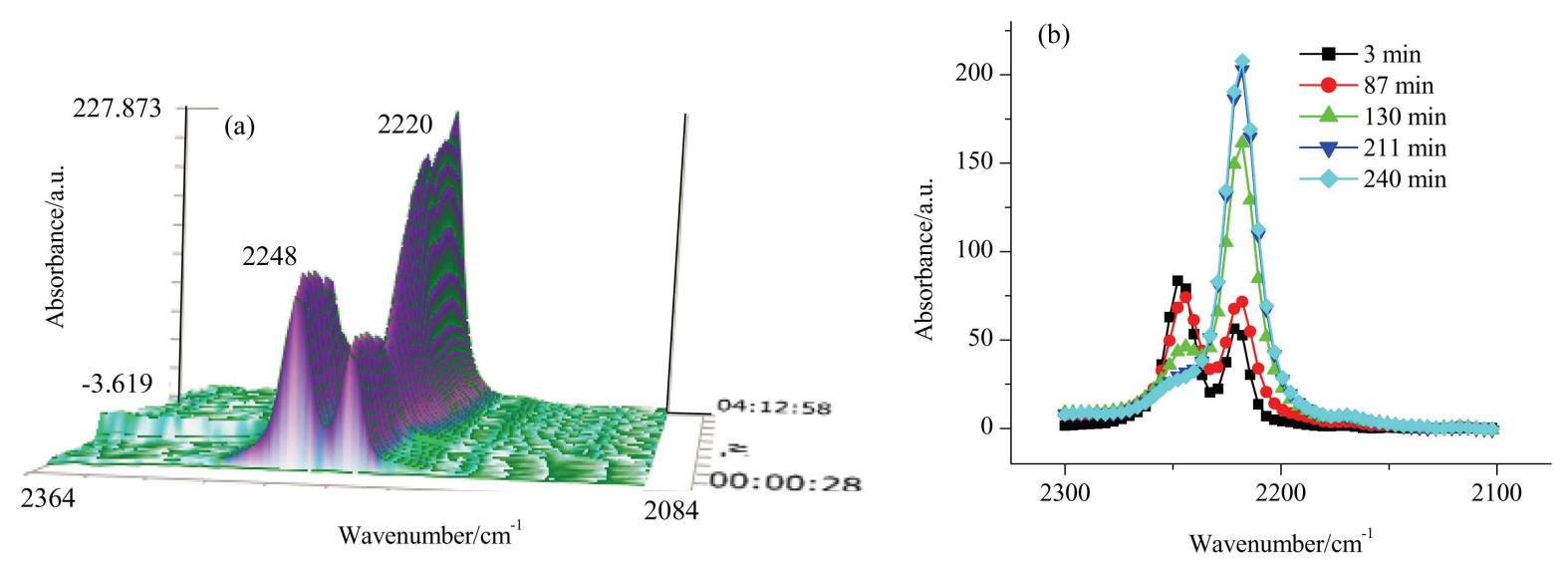

图 1 在线红外监测 $3 \mathrm{PN}$ 在零价镍催化体系中的变化情况

Figure 1 FTIR-ATR monitored variation of $3 \mathrm{PN}$ in the Ni(0) catalytic system

(a) Variation of 2248 and $2230 \mathrm{~cm}^{-1}$ absorption spectra in the process of reaction; (b) 2248 and $2230 \mathrm{~cm}^{-1}$ absorption spectra at different time

\section{References}

[1] Van Leeuwen, P. W. N. M. Homogeneous Catalysis, Academic Publisher, London, 2004, p. 229.

[2] Casalnuovo, A. L.; McKinney, R. J.; Tolman, C. A. Encyclopedia of Inorganic Chemistry, Ed.: King. R. B., Wiley, London, 1994, p. 1428 .

[3] Backvall, J. E.; Andell, O. S. Organometallics 1986, 5, 2350.

[4] (a) Tolman, C. A.; McKinney, R. J.; Seidel, W. C.; Druliner, J. D.; Stevens, W. R. Adv. Catal. 1985, 33, 1.

(b) Tolman, C. A. J. Chem. Educ. 1986, 63, 199.

[5] Druliner, J. D. Organometallics 1984, 3, 205.

[6] Crabtree, R. H. The Organometallic Chemistry of the Transition Metals, 3rd ed., Wiley, USA, 2001, p. 244.

[7] McKinney, A. R. J. Organometallics 1985, 4, 1142.

[8] William, C. D.; Tolman, C. A. Ann. N. Y. Acad. Sci. 1983, 415, 202.

[9] Van der Vlugt, J. I.; Hewat, A. C.; Neto, S.; Sablong, R.; Mills, A. M.; Lutz, M.; Spek, A. L.; Müller, C.; Vogt, D. Adv. Synth. Catal. 2004, 346, 993.

[10] Chaumonnot, A.; Lamy, F.; Etienne, S. S.; Donnadieu, B.; Chaudret, B.; Barthelat, J. C.; Galland, J. C. Organometallics 2004, 23, 3363.

[11] Bartsch, M.; Baumann, R.; Kunsmann-Keitel, D. P.; Haderlein, G.; Jungkamp, T.; Altmayer, M.; Siegel, W.; Molnar, F. DE 10150286, 2003 [Chem. Abstr. 2005, 138, 304408].

[12] Foo, T.; Garner, J. M.; Tam, W. WO 99/06357, 1999 [Chem. Abstr. 1999, 130, 169815].
[13] Alberto, A. R.; Miguel, M. H.; William, D. J.; Juventino, J. G. J. Organomet. Chem. 2006, 691, 3895.

[14] Vallée, C.; Valério, C.; Chauvin, Y.; Niccolai, G. P.; Basset, J. M.; Sautini, C. C.; Galland, J. C.; Didillon, B. J. Mol. Catal. A: Chem. 2004, 214, 71 .

[15] (a) Alberto, A. R.; Miguel, M. H.; William, D. J. J. Org. Chem. 2006, 691, 3895.

(b) Albert, A. R.; Areli, F. G.; Miguel, M. H. Organometallics 2007, 26, 1712 .

[16] Tolman, C. A.; Seidel, W. C.; Druliner, J. D.; Druliner, J. D. Organometallics 1984, 3, 33.

[17] (a) Tolman, C. A. J. Am. Chem. Soc. 1970, 92, 2956.

(b) Tolman, C. A. Chem. Rev. 1977, 77, 313.

[18] Jamer, K. P. C.; Van Leeuwen, P. W. N. M.; Reek, J. N. H. Acc. Chem. Res. 2001, 34, 895.

[19] (a) Schunn, R. A. Inorg. Synth. 1974, 15, 5. (b) Bini, L.; Müller, C.; Vogt, D. Chem. Commun. 2010, 46, 8325.

[20] Liu, Q.-B.; Huo, G.-F.; Tong, J.; Hu, X.-N. Chem. Ind. Eng. Prog. 2009, 28, 832 (in Chinese).

(刘启波, 霍光飞, 佟健, 胡晓宁, 化工进展, 2009, 28, 832.)

[21] Thomas, F.; Kristjanstdottir, S. S.; McKinney, R. J. WO 2008008929, 2008 [Chem. Abstr. 2008, 148, 146968].

[22] Wilting, J. B. M. Ph.D. Dissertation, Technische Universiteit Eindhoven, Eindhoven, Holland, 2006, Chapter 3, p. 48.

[23] Bini, L.; Houben, E. J. E.; Pidko, E. A.; Müller, C.; Vogt, D. Catal. Today 2010, 155, 271.

[24] Kitson, R. E.; Griffith, N. E. Anal. Chem. 1952, 24, 334.

(Qin, X.) 


\title{
辅助材料(Supporting Information)
}

\section{3-戊烯腈在零价镍催化体系中的平衡反应}

\author{
董建勋 ${ }^{a, b}$ 石玉坤 $^{a}$ 胡晓静 $^{a}$ 冯晓燕 ${ }^{b}$ 宋娟娟 ${ }^{a}$ 丁真贞 ${ }^{a}$ \\ 刘向明 $a^{a}$ 黄唯平 $*, a$ \\ ( ${ }^{a}$ 南开大学化学系 天津 300071) \\ ( ${ }^{b}$ 中国平煤神马能源化工集团尼龙化工公司 平顶山 467013)
}

$3 \mathrm{PN}$ 在零价镍催化体系中样品的气相色谱和质谱分析结果: 图 1,2 分别是标样和样品气相色谱分析谱图, 附图 $3 \sim 7$ 是质谱分析谱图.

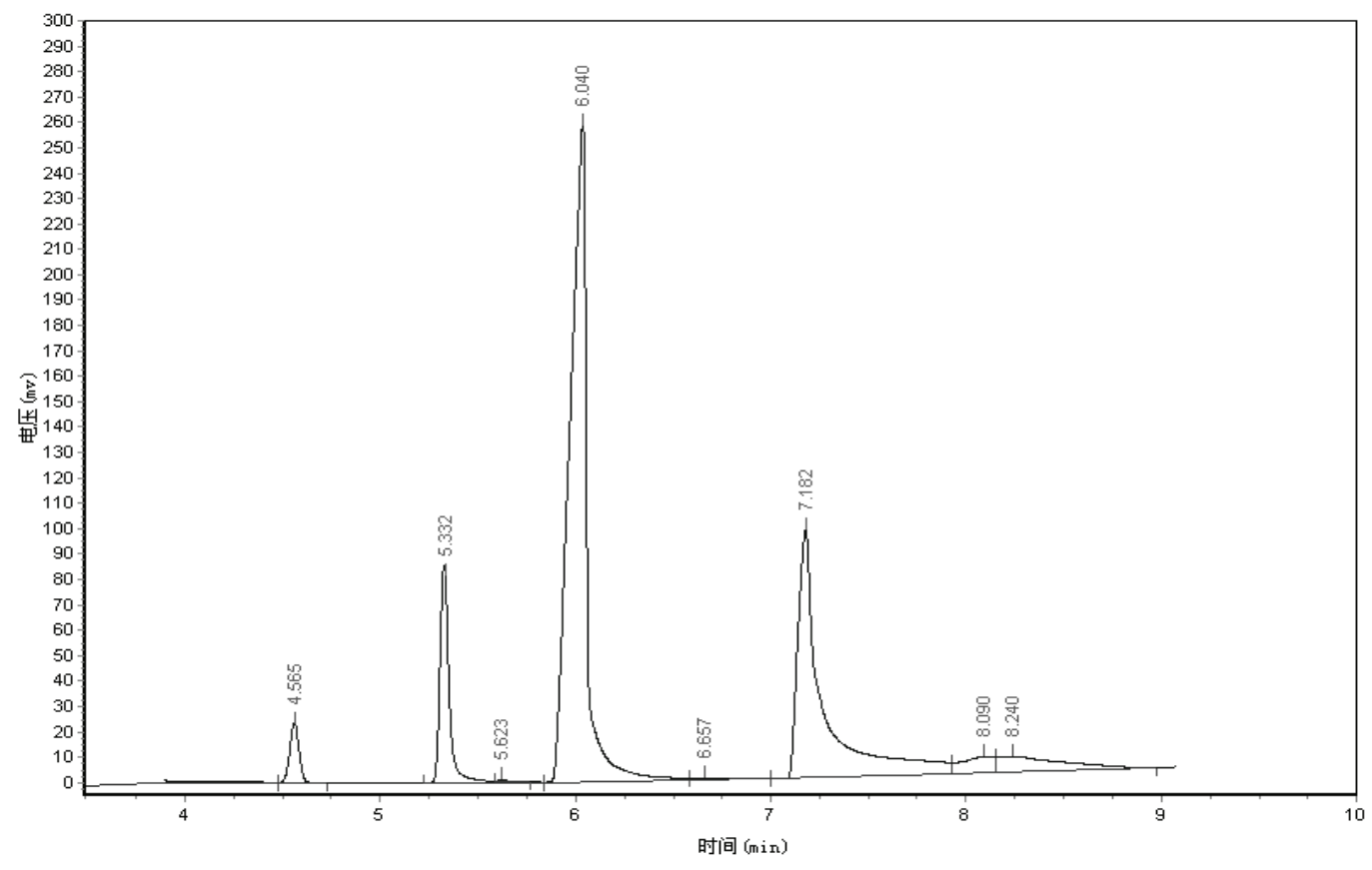

图S1 标样色谱分析图

Figure S1 GC spectrum of normal sample Retention time: $4.565 \mathrm{~min}$ for Nonane, $5.332 \mathrm{~min}$ for BN, $6.040 \mathrm{~min}$ for MGN, and $7.182 \mathrm{~min}$ for ADN 


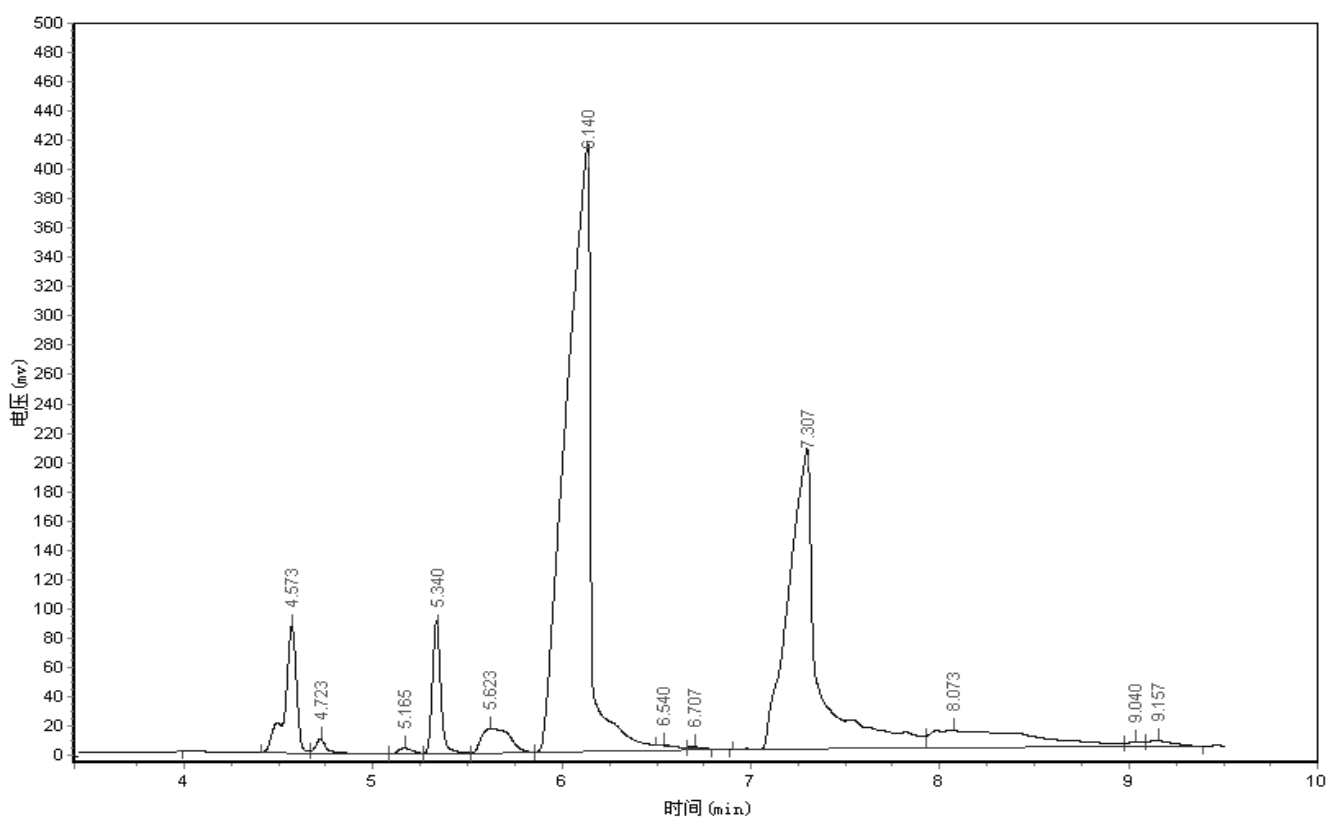

图S2 3PN在零价镍催化体系中样品色谱分析图

Figure S2 GC spectrum of sample with 3PN in zero nickel catalytic system Retention time: $4.573 \mathrm{~min}$ for Nonane, $5.340 \mathrm{~min}$ for BN, $6.140 \mathrm{~min}$ for MGN, and $7.307 \mathrm{~min}$ for ADN

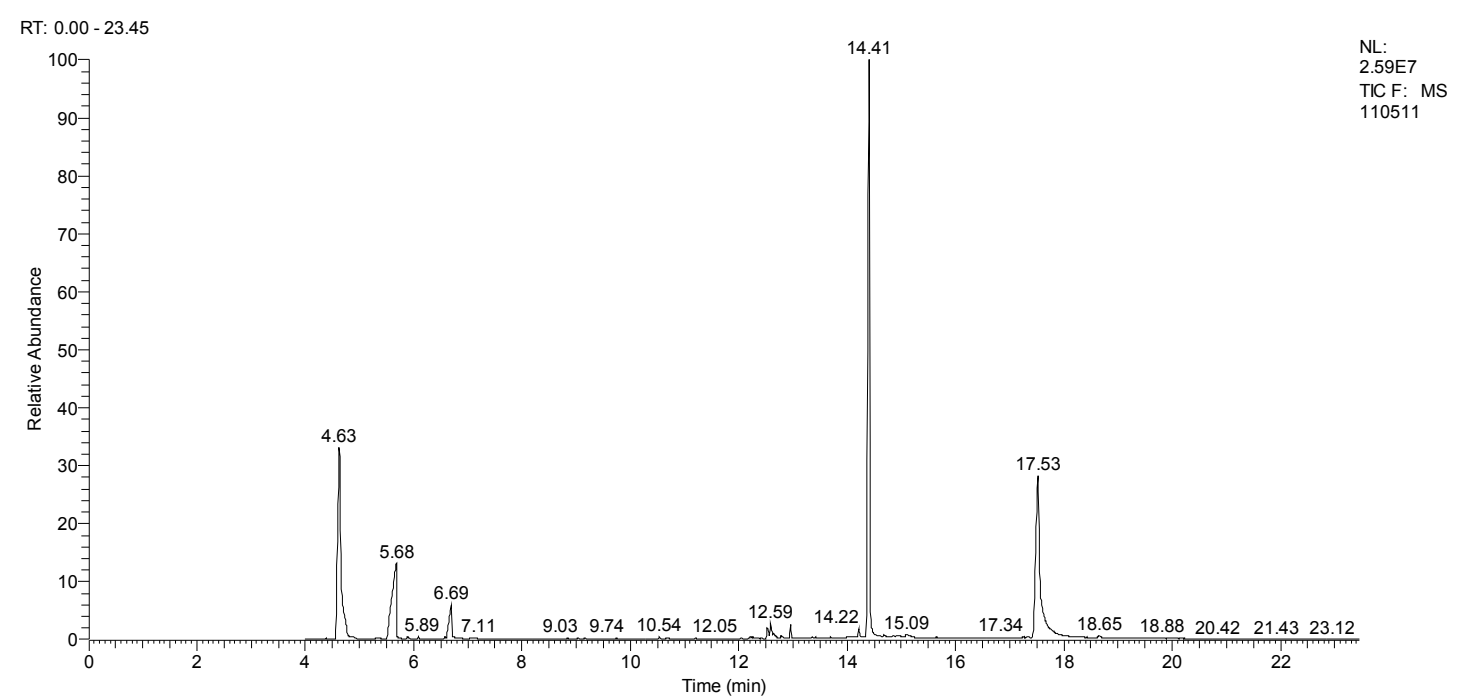

图 S3 3PN 在零价镍催化体系中样品(110511)质谱离子总图(3PN RT =3 min, 已扣除)

Figure S3 Mass spectrum total ions of sample with $3 \mathrm{PN}$ in zero nickel catalytic system (3PN RT $=3$ min, taken out) 


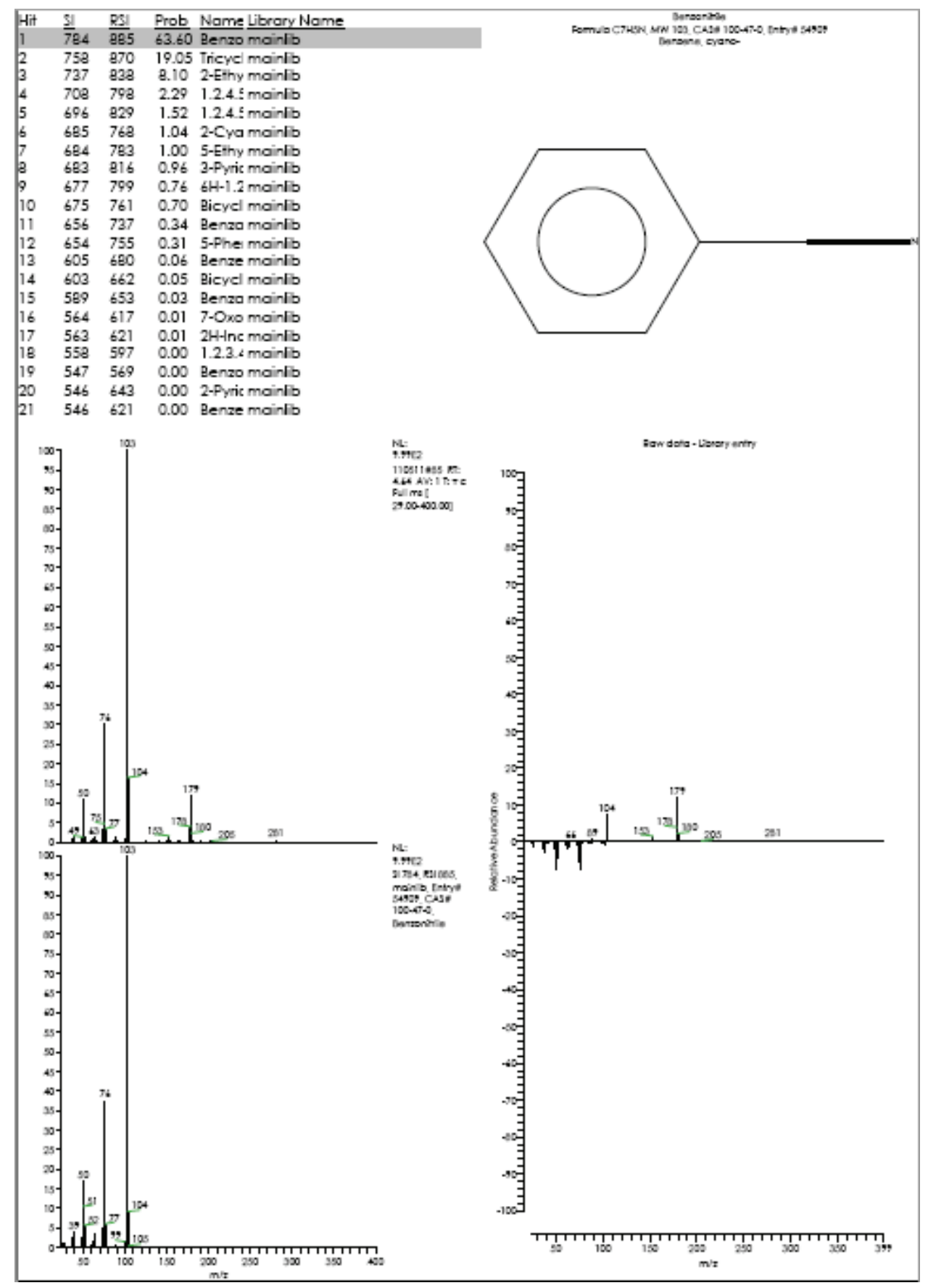

图 S4 标准图库检索 3PN 在零价镍催化体系中样品的保留时间为 $4.63 \mathrm{~min}$ 是苯甲腈, 并用纯品确认

Figure S4 Mass spectrum of BN (retention time in $4.63 \mathrm{~min}$ )is compared with standard mass spectrum map 


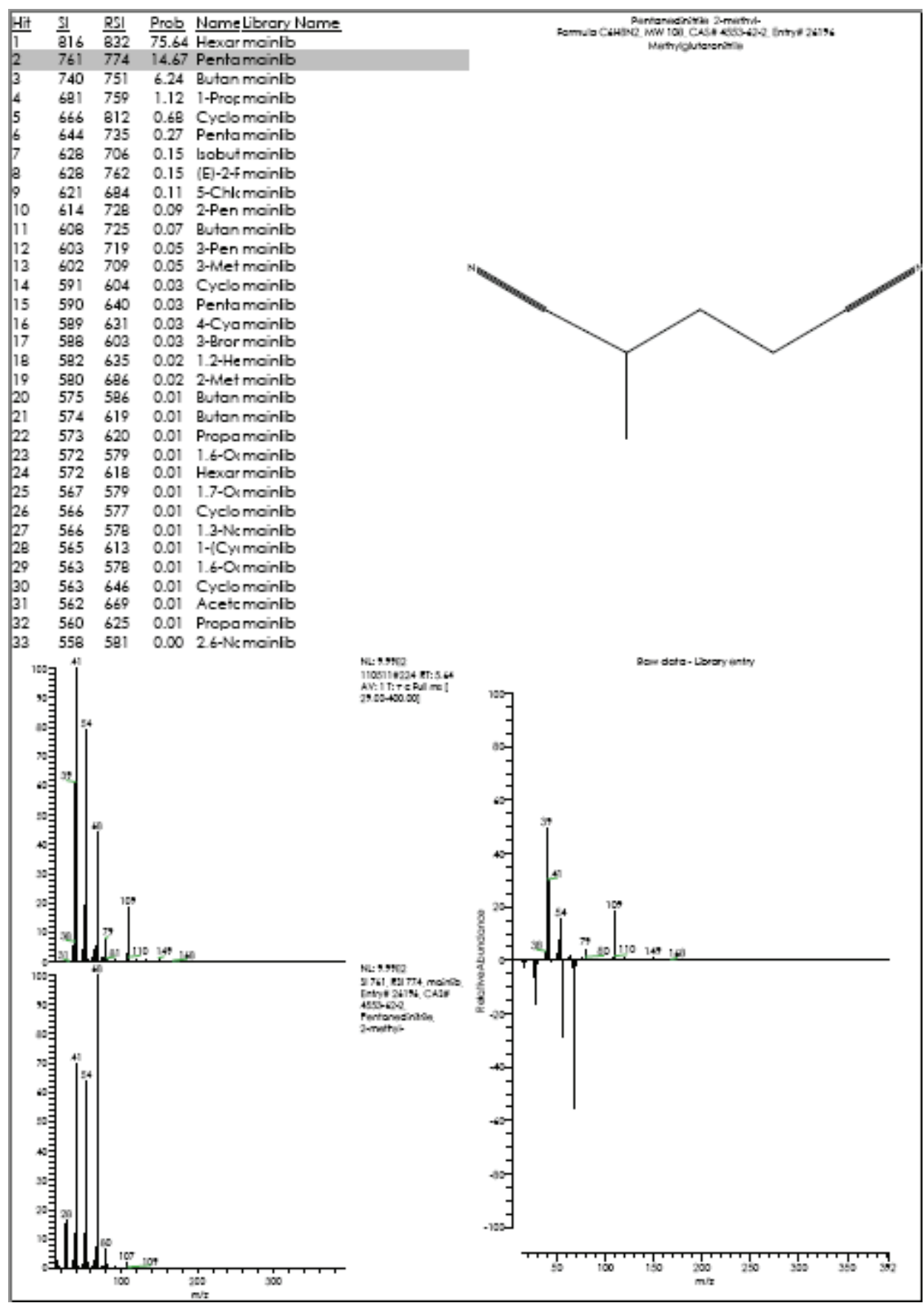

图 S5 标准图库检索 3PN 在零价镍催化体系中样品保留时间为 $5.68 \mathrm{~min}$ 的是 2-甲基戊二腈(MGN), 并用纯品确认

Figure S5 Mass spectrum of MGN (retention time in $5.68 \mathrm{~min}$ ) is compared with standard mass spectrum map 


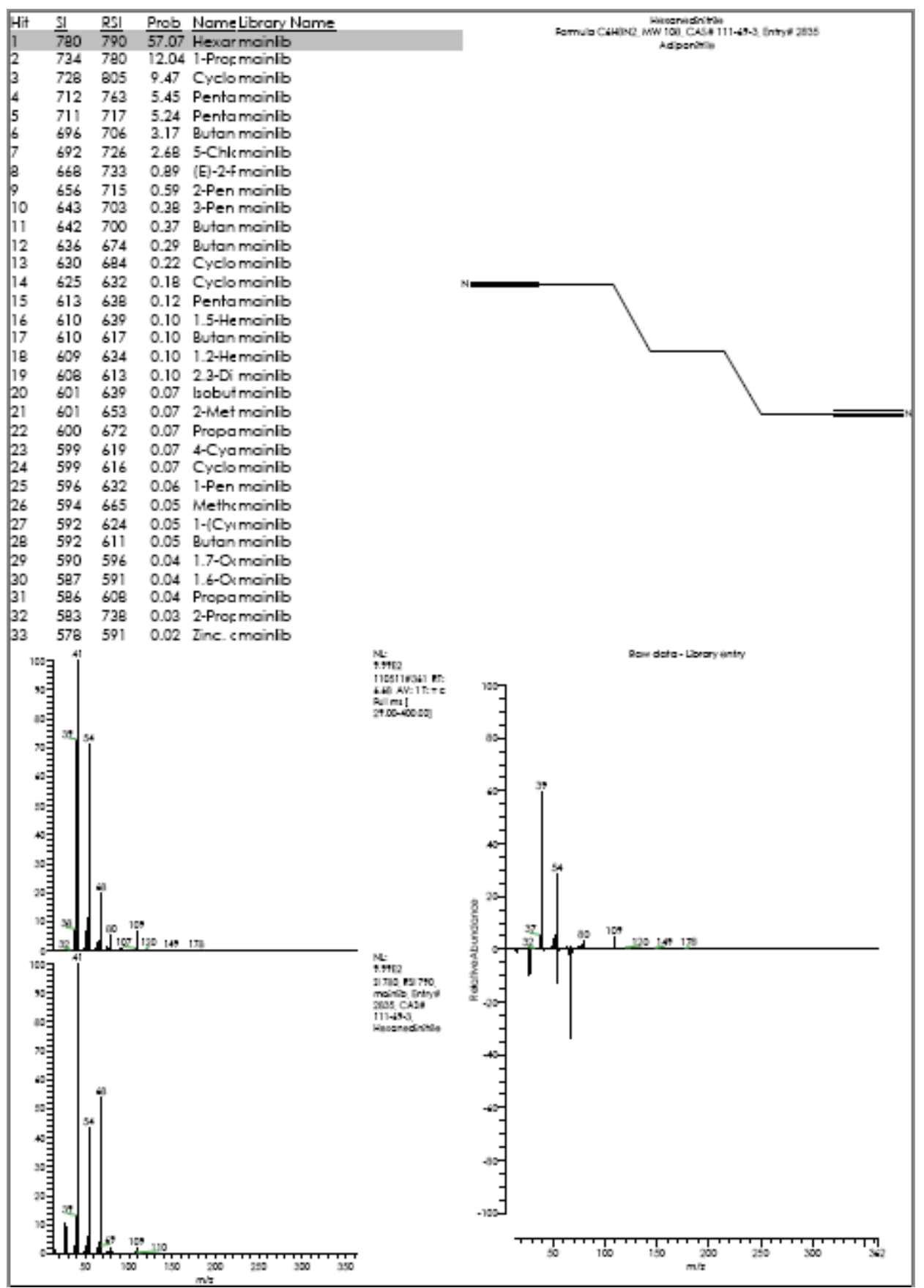

图 S6 标准图库检索 3PN 在零价镍催化体系中样品保留时间为 $6.69 \mathrm{~min}$ 是已二腈(ADN), 并用纯品确认 Figure S6 Mass spectrum of ADN (retention time in $6.69 \mathrm{~min}$ ) is compared with standard mass spectrum map 


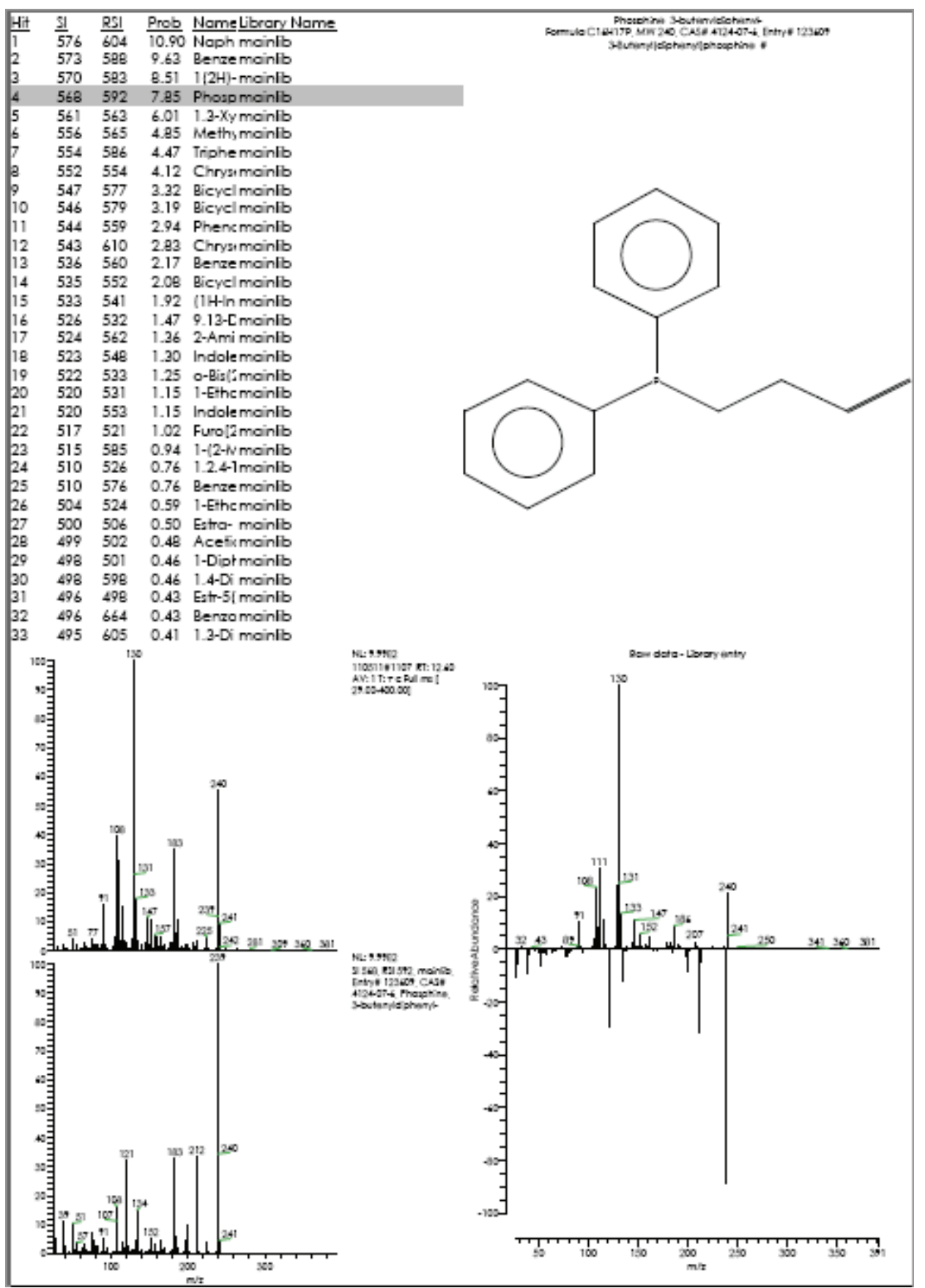

图 S7 标准图库检索 3PN 在零价镍催化体系中样品保留时间为 $12.69 \mathrm{~min}$ 是 3-丁烯基二苯基膦

Figure S7 Mass spectrum of 3-butenyl-diphenylphosphine (retention time in $12.59 \mathrm{~min}$ ) is compared with standard mass spectrum map 